\title{
Absolute fixed point sets and AR-spaces
}

by

John R. Martin * (Saskatoon)

Abstract. A subset $A$ of a topological space $X$ is a fixed point set of $X$ if there is a map (continuous function) $f: X \rightarrow X$ such that $f(x)=x$ iff $x \in A$. A compactum $X$ is an absolute fixed point set (AFS-space) if whenever $X$ is embedded in a compactum $Z$, then $X$ is a fixed point set of $Z$. In this paper it is shown that the class of AFS-spaces is properly contained in the class of compacta which are both contractible and locally contractible.

1. Introduction. The concept of an AFS-space was first defined in [2]. Clearly, every AR-space is an AFS-space. The results found in [2], [4] show that if $X$ is an AFS-space, then $X$ is a Peano continuum, and $X$ is $C^{n}$ if it is $\mathrm{LC}^{n}$ for $n=1,2, \ldots$ In [4] an example is given to show that there exists a contractible LC $^{\infty}$ compactum which is not an AFS-space.

The purpose of this paper is to show that every AFS-space is contractible and locally contractible, and to give an example of a contractible and locally contractible compactum which is not an AFS-space.

2. Notation. The diameter of a set $A$ will be denoted by $\delta(A)$. Hilbert space will be denoted by $E^{\omega}$ and given the metric $\varrho$ defined in [1, p. 10]. Definitions for the concepts of $\mathrm{LC}^{n}, \mathrm{C}^{n}, \mathrm{LC}^{\infty}, \mathrm{AR}$-space, and ANR-space may be found in [1]. We shall also make use of the concept of the cap over a continuum which we now define.

Let $C$ be a continuum lying in $E^{\omega}$ and let $A$ be a compact segment in $E^{\infty}$. Consider the disjoint union $A \cup(C \times[0, \infty))$ of $A$ and the product space $C \times[0, \infty)$. Let $h$ be a one-to-one mapping from $A \cup(C \times[0, \infty))$ onto a continuum in $E^{\infty}$ such that the following properties are satisfied.

(1) $h(z)=z$ if $z \in A$.

(2) $h(z, 0)=z$ for all $z \in C$.

(3) $h \mid C \times[0, \infty)$ is a homeomorphism.

(4) $\lim _{t \rightarrow \infty} \delta(h(C \times\{t\}))=0$

- The research for this article was supported in part by the National Research Council of Canada (Grand A8205) 
(5) For each $z \in C, h(\{z\} \times[0, \infty)) \cup A$ is homeomorphic to the closure of the curve in the plane $E^{2}$ whose equation is $y=\sin (\pi / x)$ for $0<x \leqslant 1$.

The image of $A \cup(C \times[0, \infty))$ under $h$ is defined to be the cap $C A$ of $C$ and $A$. We shall regard the cap $C A$ as the set $A \cup(C \times[0, \infty))$ together with an assigned metric which makes the function $h$ an isometry.

Essentially, the cap $C A$ is obtained by taking a cone over $C$, removing the vertex, and then winding the resulting cylinder over $C$ in a " $\sin (1 / x)$ " fashion in $E^{\omega}$ so as to converge to the limit interval $A$.

3. The results. The proof of the following theorem parallels the proof of Property 3 in [3, p. 165].

THeOREM 1. Every locally contractible AFS-space is contractible.

Proof. Suppose that $X$ is a locally contractible AFS-space which is not contractible. Let $A$ denote the closed interval $[-1,1]$, and let $Z$ denote the cap $X A$ We shall consider $X$ to be identified with $X \times\{0\}$ in $Z$. It follows that $Z-A$ is locally contractible, and $X$ is not contractible in $Z$.

There is a map $f: Z \rightarrow Z$ whose fixed point set is precisely $X$. Since $A$ has the fixed point property, it follows that there is a point $p$ in $A$ such that $f(p) \in Z-A$. Let $V$ be a neighborhood of $f(p)$ which is contractible in $Z-A$, and let $\beta: V \times I \rightarrow Z-A$ denote a homotopy which deforms $V$ to a point. Since $f$ is continuous at $p$, there is a neighborhood $U$ of $p$ in $Z$ such that $f(U) \subset V$. Then, for some $q$ in $[0, \infty)$, we have $X \times\{q\} \subset U$. Let $\alpha: X \times I \rightarrow Z-A$ denote a homotopy which deforms $X$ onto $X \times\{q\}$. Define a homotopy $H: X \times I \rightarrow Z$ by

$$
H(x, t)= \begin{cases}f(\alpha(x, 2 t)), & \text { (if } \left.0 \leqslant t \leqslant \frac{1}{2}\right), \\ \beta(f(\alpha(x, 1)), 2 t-1), & \text { (if } \left.\frac{1}{2} \leqslant t \leqslant 1\right) .\end{cases}
$$

It is easy to check that $H$ is a homotopy which deforms $X$ to a point in $Z$. This contradiction shows that $X$ must be contractible.

COROLlary. $A$ compactum $X$ is an AR-space iff $X$ is an AFS-space and $X$ is an ANR-space.

Proof. Suppose that $X$ is an ANR-space and an AFS-space. Since every ANR -space is locally contractible, it follows from Theorem 1 that $X$ is a contractible ANR-space, and hence an AR-space $[1, p .101]$. This completes the proof since every AR-space is both an AFS-space and an ANR-space.

We remark that Proposition 1 in [4] shows that if $X$ is an AFS-space which is $\mathrm{LC}^{n}$, then $X$ is $\mathrm{C}^{n}$ for $n=0,1,2, \ldots$ It follows that (see $[1, \mathrm{p} .122]$ ), for the case of finite dimensional AFS-spaces, Theorem 1 is a consequence of Proposition 1 of [4]

THEOREM 2. Every AFS-space is locally contractible.

Proof. Let $X$ be an AFS-space and suppose that $X$ is not locally contractible at a point $p$. Then there is a neighborhood $U$ of $p$ in $X$ which contains a decreasing sequence $V_{1}, V_{2}, \ldots$ of compact neighborhoods of $p$ such that $\lim \delta\left(V_{i}\right)=0$ and no $V_{i}$ is contractible in $U$. Let $I$ denote the closed unit interval $[0,1]$, and let $A_{i}=[1 / 2 i, 1 /(2 i-1)]$ for $i=1,2, \ldots$ Consider a sequence of disjoint continua $Y_{1}, Y_{2}, \ldots$ with $\lim _{i \rightarrow \infty} \delta\left(Y_{i}\right)=0$ obtained by first taking the disjoint union $\bigcup_{i=1}^{\infty} V_{i}$ and then letting $Y_{i}=\operatorname{cap} V_{i} A_{i}$ for $i=1,2, \ldots$ Let $Y=I \cup \bigcup_{i=1}^{\infty} Y_{i}$. Form the compactum $Z_{1}$ obtained by taking the disjoint union $X \cup Y$ and then identifying $V$ in $X$ with $V_{i} \times\{0\}$ in $Y$ for $i=1,2, \ldots$, and identifying the point $p$ in $X$ with the point 0 in $I$.

Now suppose $f_{1}: Z_{1} \rightarrow Z_{1}$ is a map whose fixed point set is precisely $X$. First we show that there are infinitely many sets of the form $f_{1}\left(A_{i}\right)$ such that $f_{1}\left(A_{i}\right) \cap(0,1]=\varnothing$. To see this, suppose $f_{1}\left(A_{i}\right) \cap(0,1] \neq \varnothing$ for all but finitely many of the sets $f_{1}\left(A_{i}\right), i=1,2, \ldots$ Let $W$ be a neighborhood of $p$ in $Z_{1}$ such that $W \cap X=U$. Since $f_{1}$ is continuous at $p$, there is a neighborhood $V$ of $p$ in $Z_{1}$ such that $V \subset W$ and $f_{1}(V) \subset W$. Now let $k$ be a positive integer such that $Y_{i} \subset V$ and $f_{1}\left(A_{k}\right) \cap(0,1] \neq \varnothing$

Suppose there is a point $a$ in $A_{k}$ and a neighborhood $M$ of $a$ in $W$ such that $f_{1}(M) \subset I$. Then, for some $t_{1}>0, f_{1}\left(V_{k} \times\left\{t_{1}\right\}\right) \subset I$. Since $f_{1}$ is the identity on $V_{k}=V_{k} \times\{0\}$ and $V_{k}$ can be deformed onto $V_{k} \times\left\{t_{1}\right\}$ in $Y_{k}$, it follows that $V_{k}$ is contractible in $f_{1}\left(Y_{k}\right)$. Let $r: f_{1}\left(Y_{k}\right) \rightarrow f_{1}\left(Y_{k}\right) \cap X$ be a retraction defined by

$$
r(y)=\left\{\begin{array}{lll}
p & \text { if } & y \in f_{1}\left(Y_{k}\right) \cap I, \\
y & \text { if } & y \in f_{1}\left(Y_{k}\right) \cap X, \\
(x, 0) & \text { if } & y=(x, t) \in f_{1}\left(Y_{k}\right) \cap\left(\bigcup_{i=1}^{\infty} Y_{i}\right) .
\end{array}\right.
$$

It then follows that $V_{k}$ is contractible in $r\left(f_{1}\left(Y_{k}\right)\right) \subset W \cap X=U$. This contradiction shows that no neighborhood $M$ of a point $a$ in $A_{k}$ has the property that $f_{1}(M) \subset I$.

Now let $N$ be a positive integer such that if $i>N$, then $Y_{i} \subset V$ and $f_{1}\left(A_{i}\right) \cap(0,1] \neq \varnothing$. It follows from the above argument that if $i>N$, then $f_{1}\left(A_{i}\right)=A_{j}$ for some $j$. Moreover, since 0 is the only point in $I$ which remains fixed under $f_{1}$, it is easy to show that if $x, f_{1}(x) \in I$, then $f_{1}(x)<x$. Consequently, if $i>N$, we have $f_{1}\left(A_{i}\right)=A_{j}$ for some $j>i$.

Define $\sigma(0)=N+1$ and, if $m$ is a positive integer, let $\sigma(m)$ denote the unique positive integer satisfying the equation $f_{1}\left(A_{\sigma(m-1)}\right)=A_{\sigma(m)}$. Since $f_{1}\left(A_{N+1}\right)=A_{\sigma(1)}$, it follows that, for some $t_{2}>0, f_{1}\left(V_{N+1} \times\left\{t_{2}\right\}\right) \subset Y_{\sigma(1)} \cap(Z-(0,1])$. Then, as in (*), we may define a retraction

$$
s: f_{1}\left(V_{N+1} \times\left[0, t_{2}\right]\right) \rightarrow f_{1}\left(V_{N+1} \times\left[0, t_{2}\right]\right) \cap X .
$$

Since $V_{N+1}=V_{N+1} \times\{0\}$ is homotopic to $V_{N+1} \times\left\{t_{2}\right\}$ in $V_{N+1} \times\left[0, t_{2}\right]$, it follows that $C_{1}=V_{N+1}$ is homotopic to $C_{2}=s f_{1}\left(V_{N+1} \times\left\{t_{2}\right\}\right)$ under a homotopy $H_{1}$ whose image, $\operatorname{Im} H_{1}$, lies in $U$. This argument can be repeated to obtain a sequence of sets $C_{1}, C_{2}, \ldots, C_{i}, \ldots$ in $X$ and a corresponding sequence $H_{1}, H_{2}, \ldots, H_{i}, \ldots$ of homotopies with values in $U$ such that the following properties are satisfied. - Fundamenta Mathematicae CXII 
(1) $C_{i} \subset V_{\sigma(i-1)} \times\{0\}$ for $i=1,2, \ldots$

(2) $H_{i}: C_{i} \times I \rightarrow U$ with $H_{i}\left(C_{i} \times\{0\}\right)=C_{i}$ and $H_{i}\left(C_{i} \times\{1\}\right)=C_{i+1}$ for $i=1,2, \ldots$

(3) $\lim _{i \rightarrow \infty} \delta\left(H_{i}\left(C_{i} \times I\right)\right)=0$.

It is possible to construct a homotypy $H: C_{1} \times I \rightarrow U$ such that, for $i=1,2, \ldots$ $H\left(C_{1} \times\{(i-1) / i\}\right)=C_{i}$ and $H\left(C_{1} \times\{1\}\right)=p$. This contradicts the fact that $C_{1}$, $=V_{N+1}$ is not contractible in $U$. Consequently, for infinitely many of the integers $i=1,2, \ldots, f_{1}\left(A_{i}\right) \cap(0,1]=\emptyset$.

Let $Q=\bigcup\left\{Y_{i} \mid f_{1}\left(A_{i}\right) \cap(0,1]=\varnothing\right\}$. Then, as in (*), we may define a retraction $r_{1}: f_{1}(X \cup Q) \rightarrow X$. Let

$$
Q_{1}=U\left\{Y_{i} \mid Y_{i} \subset Q \text { and } r_{1} f_{1}\left(Y_{i}\right) \subset U\right\} \text {. }
$$

Henceforth, we shall only consider the subsequence $Y_{i_{1}}, Y_{i_{2}}, \ldots$ of $\left\{Y_{i}\right\}_{i=1}^{\infty}$ such that $Q_{1}=\bigcup_{j=1}^{\infty} Y_{i_{j}}$. To facilitate notation we shall relabel this subsequence $Y_{1}, Y_{2}, \ldots$ Since $r_{1} f_{1}\left(Y_{i}\right) \subset U$, it follows that $\delta\left(r_{1} f_{1}\left(A_{i}\right)\right) \neq 0$ for $i=1,2, \ldots$ Otherwise, some $V_{j}$ would be contractible in $U$ which is not possible. Thus, we may assume that $\delta\left(r_{1} f_{1}\left(A_{i}\right)\right)=\lambda_{i}$ where $\lambda_{1}, \lambda_{2}, \ldots$ is a sequence of positive numbers which converges to 0 .

Let $a_{i}$ denote the midpoint of $A_{i}$, and let $0=t_{i, 0}<t_{i, 1}<\ldots<t_{i, j}<\ldots$ be a sequence of numbers in $[0, \infty)$ such that $\lim V_{i} \times\left\{t_{i, j}\right\}=a_{i}$. Set $b_{i}=r_{1} f_{1}\left(a_{i}\right)$, $B_{i, j}=r_{1} f_{1}\left(V_{i} \times\left\{t_{i, j}\right\}\right)$, and let $Y_{i, j}=\operatorname{cap}\left(B_{i, j}^{j \rightarrow \infty} A_{i, j}\right)$ denote a null sequence of caps such that $\lim _{j \rightarrow \infty} Y_{i, j}=b_{i}$. Let $\left\{I_{i}\right\}_{i=1}^{\infty}$ be a null sequence of arcs such that $I_{i} \cap X=\left\{b_{i}\right\}$ and, for all $j=1,2, \ldots, A_{i, j} \subset I_{i}$. Form the compactum $Z_{2}=X \cup \bigcup_{i, j=1}^{\infty}\left(Y_{i, j} \cup I_{i}\right)$.

Since $X$ is an AFS-space, there is a map $f_{2}: Z_{2} \rightarrow Z_{2}$ whose fixed point set is precisely $X$. Then, using arguments similar to previous arguments, there exists a set $Q_{2}$ which is the union of infinitely many sets of the form $Y_{i, j}$, and there exists a retraction $r_{2}: f_{2}\left(X \cup Q_{2}\right) \rightarrow X$ such that $r_{2} f_{2}\left(Y_{i, j}\right) \subset U$ for each $Y_{i, j} \subset Q_{2}$. The process can be continued so that in the $n$th stage we obtain a retraction $r_{n}: f_{n}\left(X \cup Q_{n}\right) \rightarrow X$ such that $r_{n} f_{n}\left(Y_{i_{1}, i_{2}, \ldots, i_{n}}\right) \subset U$ for each of the $Y_{i_{1}, i_{2}, \ldots, i_{n}}$ lying in $Q_{n}$. In particular, there is a sequence $V_{j_{1}}=B_{j_{1}, 0}, B_{j_{1}, j_{2}}, B_{j_{1}, j_{2}, j_{3}}, \ldots, B_{j_{1}, j_{2}, \ldots, j_{n}}, \ldots$ of sets lying in $U$, a point $q \in U$, and a sequence of homotopies $\left\{F_{n}\right\}_{n=1}^{\infty}$ with values in $U$ such that the following properties are satisfied.

(1) $\lim _{n \rightarrow \infty} B_{j_{1}, j_{2}, \ldots, j_{n}}=\{q\}$.

(2) $F_{1}$ is a homotopy which deforms $V_{j_{1}}$ onto $B_{j_{1}, j_{2}}$ and, for $n>1, F_{n}$ deforms $B_{j_{1}, j_{2}, \ldots, j_{n}}$ onto $B_{j_{1}, j_{2}, \ldots, j_{n, j_{n+1}}}$.

$$
\text { (3) } \lim _{n \rightarrow \infty} \delta\left(\operatorname{Im} F_{n}\right)=0 \text {. }
$$

It is possible to construct a homotopy $F$ with values in $U$ which deforms $V_{J_{1}}$ to the point $q$. This contradiction shows that $X$ must be locally contractible as required.
Theorems 1 and 2 show that every AFS-space is contractible and locally contractible. Thus every finite dimensional AFS-space is an AR-space [1, p. 122] Since every AR-space is an AFS-space, we have the following theorem.

THEOREM 3. A finite dimensional compactum $X$ is an AFS-space iff $X$ is an AR-space.

The following result was pointed out to the author by E. D. Tymchatyn. Since a retract of an AR-space is an AR-space [1, p. 101], the finite dimensional case follows from Theorem 3 .

Proposition. Every retract of an AFS-space is an AFS-space.

Proof. Let $X$ be a retract of an AFS-space $Y$, and let $e: X \rightarrow Z$ be an embedding of $X$ into a compactum $Z$. Let $W$ be the identification space obtained by taking the disjoint union of $Y$ and $Z$, and then identifying each $x \in X$ with $e(x) \in Z$. We shall regard $X, Y, Z$ as subspaces of the compactum $W$. Since $Y$ is an AFS-space, there is a mapping $g: W \rightarrow W$ whose fixed point set is precisely $Y$. Let $r: Y \rightarrow X$ be a retraction of $Y$ onto $\dot{X}$. Define a function $f: Z \rightarrow Z$ by

$$
f(z)=\left\{\begin{array}{lll}
g(z) & \text { if } & g(z) \in Z, \\
r g(z) & \text { if } & g(z) \in Y-X .
\end{array}\right.
$$

It is easy to check that $f$ is a mapping whose fixed point set is precisely $X$.

An example of a contractible LC ${ }^{\infty}$ compactum which is not an AFS-space is given in [4]. We now use similar techniques to those found in [4] to show that a wellknown example due to Borsuk [1, p. 126] is in fact an example of a contractible and locally contractible compactum which is not an AFS-space.

EXAMPLE. Consider the following subsets of the Hilbert cube $Q^{\infty}$ (for notation see $[1$, p. 10]):

$$
\begin{aligned}
X_{0} & =\left\{x=\left\{x_{i}\right\} \mid x_{1}=0\right\}, \\
B_{k} & =\left\{x=\left\{x_{i}\right\} \mid 1 /(k+1) \leqslant x_{1} \leqslant 1 / k \text { and } x_{i}=0 \text { for } i>k\right\} \text { for } k=1,2, \ldots
\end{aligned}
$$

The boundary $\mathrm{Bd} B_{k}$ of $B_{k}$ is a $(k-1)$-sphere which we shall denote by $X_{k}$ for $k=1,2, \ldots$ Let $X=X_{0} \cup \bigcup_{k=1}^{\infty} X_{k}$. Then, if $Y$ denotes the cone over $X$ with vertex $p$, $Y$ is a contractible and locally contractible compactum which is not an ANR-space $[1$, p. 126]. We now show that $Y$ is not an AFS-space by constructing a compactum $Z$ containing $Y$ such that $Y$ is not a fixed point set of $Z$.

Let $C_{k}=\operatorname{cap} X_{k} A_{k}, k=1,2, \ldots$, denote a sequence of caps in Hilbert space $E^{c}$ such that the following properties are satisfied.

(1) $C_{k} \cap Y=X_{k} \times\{0\}=X_{k}$ for $k=1,2, \ldots$

(2) $C_{i} \cap C_{j}=X_{i} \cap X_{j}$ for $i, j=1,2, \ldots, i \neq j$.

(3) $\lim _{k \rightarrow \infty} C_{k}=X_{0}$.

Define $Z=Y \cup \bigcup_{k=1}^{\infty} C_{k}$. 
Suppose that $f: Z \rightarrow Z$ is a mapping whose fixed point set is precisely $Y$. Since $\lim _{k \rightarrow \infty} C_{k}=X_{0}$ and $f\left(X_{0}\right)=X_{0}$, there is a positive integer $j$ such that $f\left(C_{j}\right) \subset Z-\{p\}$. Furthermore, since no point in $A_{j}$ remains fixed under $f$, it follows that $f\left(A_{j}\right) \subset Z-\left(A_{j} \cup\{p\}\right)$. Let $r$ denote a retraction which maps $Z-\left(A_{j} \cup\{p\}\right)$ onto $X_{j}$. Then $r f \mid C_{j}$ is a retraction from $C_{j}$ onto $X_{j}=X_{j} \times\{0\}$. It then follows from the proof of Theorem 1 that $X_{j}$ is contractible. This contradiction shows that $Y$ is not a fixed point set of $Z$.

We remark that the above example together with Theorems 1 and 2 show that the class of AFS-spaces is properly contained in the class of contractible and locally contractible compacta. In view of this, it seems appropriate to pose the following question.

QUESTION. Does the class of AFS-spaces coincide with the class of AR-spaces?

\section{References}

[1] K. Borsuk, Theory of Retracts, Warszawa 1967.

[2] J. R. Martin, On absolute fixed point sets, Colloq. Math. 35 (1976), pp. 67-71.

[3] - Fixed point sets of Peano continua, Pacific J. Math. 74 (1978), pp. 163-166.

[4] - Absolute fixed point sets in compacta, Colloq. Math. 39 (1978), pp. 41-44.

DEPARTMENT OF MATHEMATICS

UNIVERSITY OF SASKATCHEWAN

Saskatoon, Canada

Accepté par la Rédaction le 19. 2. 1979 